\title{
DORSAL HAND VEIN PATTERN AUTHENTICATION BY HOUGH PEAKS
}

\author{
V.Krishna Sree ${ }^{1}$, P.Sudhakar Rao ${ }^{2}$ \\ ${ }^{I}$ Dept of ECE, VNR VJIET, Hyderabad, India \\ ${ }^{2}$ Dept of ECE, VITS, Hyderabad, India
}

\begin{abstract}
The quest of providing more secure identification system has led to rise in developing biometric systems. Biometrics such as face, fingerprint and iris have been developed extensively for human identification purpose and also to provide authentic input to many security systems in the past few decades. Dorsal hand vein pattern is an emerging biometric which is unique to every individual. In this paper Linear Hough transform is used to extract the features of query and data base images. K-Nearest neighbor Search is used to obtain best match between query image and database. The extraction of the vein patterns was obtained by morphological techniques. Noise reduction filters are used to enhance the vein patterns.
\end{abstract}

Keywords:- Dorsal hand vein patterns, K-Nearest neighbor search, Morphological Techniques, Enhancement , Noise Reduction and Hough transform.

\section{INTRODUCTION}

Personal identification systems are gaining lot of demand due to increased threats and attacks from the terrorists. These can be prevented by tightening the security at important places. The traditional methods make use of smart cards or personal identification numbers etc to identify a person. However these methods have limited security and are unreliable. Over the past years various biometric systems have been developed to overcome these disadvantages[5]. Biometrics is the science of identifying a person using its physiological or behavioral features. These features range from physical traits like fingerprints, faces, iris etc to represent the signature or personal behavior of a human being. Compared to traditional methods biometric features are much harder for intruders to copy or forge and it has one more advantage that it is very rare for them to be lost. Hence for identification systems making use of biometric features offer a much more secure and reliable performance. Each of these biometric features has its strengths and weaknesses. Vein pattern is the network of blood vessels beneath a person's skin. This vein pattern can be used to authenticate the identity of an individual.

Anatomically aside from surgical intervention the shape of the vascular patterns in the back of the hand is distinct from each other and it remains stable over along period[2]. In addition as the blood vessels are hidden underneath the skin and are invisible to the human eye, vein patterns are much harder for intruders to copy as compared to other biometric features[9], vein image which can be taken only at live body, Due to noncontact, it is hygienic and non-duplicating and has no negative image associated with crime. All these special properties of hand vein patterns make it a potentially good biometric to offer more secure and reliable features for personal verification[1]. Biometrics is a science for uniquely recognizing humans based upon one or more intrinsic physical or behavioral traits. Biometrics is used as a form of identity access management, access control and identifying individuals in groups under surveillance. Biometrics can be divided in two main classes that are physical and behavioral. Physical are related to the shape of the body like fingerprint, face recognition, DNA, palm print, hand geometry and iris of the eye. Behavioral are related to behavior of a person like voice, gait etc. The biometrics should have the certain characteristics like each person should have the said characteristic, it should distinguish individual from another, should be resistive to ageing, easy to acquire, should be accurate, robust, and should have acceptability. A biometric which possesses more number of characteristics is treated as a good bio-metric[6].

A biometric system can operate in two modes, one is Verification and Identification. Verification is the one to one comparison of a captured biometric with a stored template to verify that the individual is who he claims to be. Can be done in conjunction with a smart card, user ID number etc. Identification is a one to many comparisons of the captured biometric against a biometric data base in attempt to identify an unknown individual. The identification only succeeds in identifying the individual if the comparison of the biometric sample to a template in the data base falls within a previously set threshold. Before the Verification or Identification an individual should enroll his information in the system to store it as template for subsequent uses. During the identification process, the Query image is matched with the set of templates available as data base 
with the help of some image processing algorithms. During this signature recognition, lot of comparisons need to be with every template in the database. Hough transform is used to identifythe featurepatterns in database as well as in the query image. KNNsearch is used to search for similarities between features of the query image and database in a speedy and effective manner. The memory requirements are reduced in KNN search which is very much needed in case of large database. In this work Hough transform in combination with $\mathrm{KNN}$ search is used for identifying the query hand vein pattern image from the data base of hand vein pattern images.

\section{MATERIALS}

Veins are hidden underneath the skin, and are invisible to the naked eye and other visual inspection systems. However human superficial veins have higher temperature than the surrounding tissue. Based on this fact, the vein pattern in the back of the hand can be captured using a thermal camera. In this work NEC Thermal tracer is utilized to acquire thermal images of the back of the hand. The images collected from differentpeople in a normal office environment between 20-25oc. One image of hand vein dorsal is shown in Fig. 1.

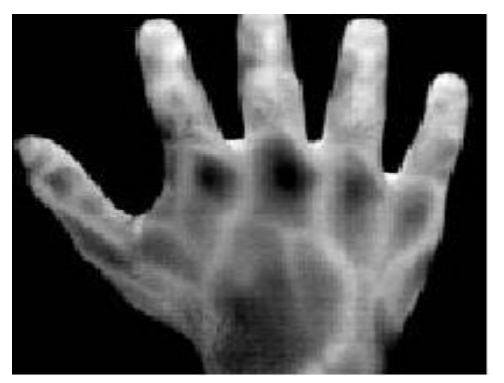

Fig.1: Thermal dorsal hand vein image

\section{METHOD}

The steps involved in the proposed work are given in Fig.2.

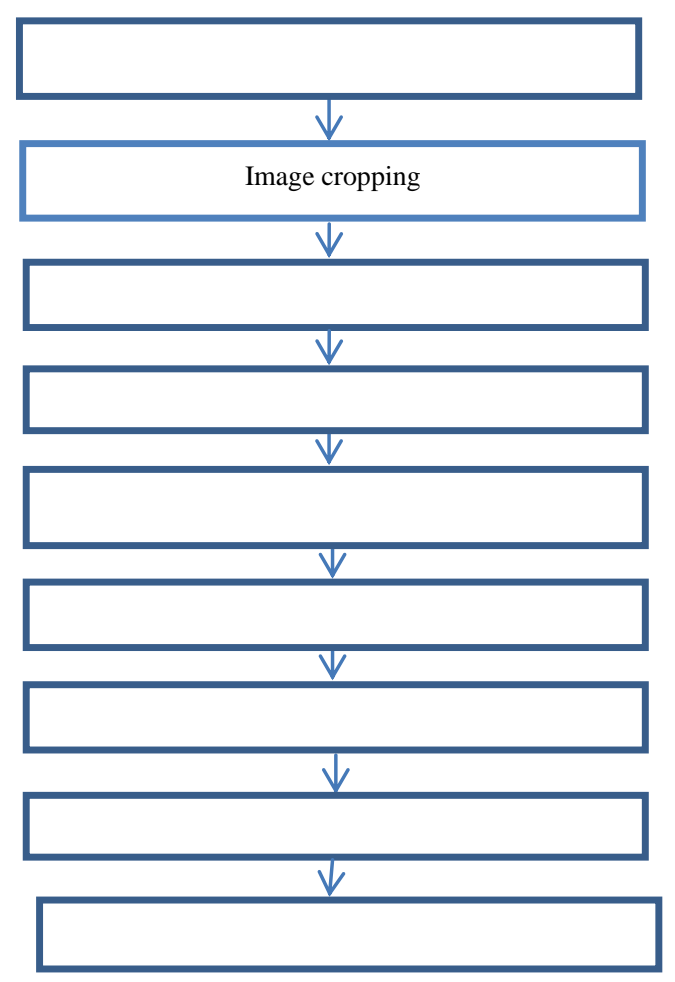

Fig.2: Steps Involved in the proposed technique

The input hand Vein pattern thermal images are cropped to extract the region of interest. The cropped images are grouped as training set. The training set is enhanced using adaptive histogram equalization. On equalized training set noise filtering is carried out to minimize the noise. Thinning is then performed to reduce the vein patterns into a pixel wide. Hough transform is applied on these thinned images to extract the Hough peaks and the corresponding radius(r) and angle $(\Theta)$ values as feature vectors. For the Query image to be verified from the database is cropped, adaptive histogram equalized,filtered and thinned. The Hough peaks and the associated radius(r) and angle $(\Theta)$ are extracted for that. And matching is performed by using KNN search.

\subsection{Preprocessing}

In preprocessing the hand vein pattern images are cropped to the required region of interest where veins look prominent. The portions of fingers are removed and to adjust the intensities uniformly adaptive histogram equalization has been carried out $[4,7]$. The collected data base is converted into training set. On this training set median filtering is applied to remove the noise. Thinning is performed as the final preprocessing step to extract one pixel wide vein patterns before the feature extraction.

\subsection{Hough Transform}

The Hough transform is a feature extraction technique used in image analysis, computer vision, and digital image processing to find imperfect instances of objects within a certain class of 
shapes circles or ellipses by a voting procedure. This voting procedure is carried out in a parameter space, from which object candidates are obtained as local maxima in a so-called accumulator space that is explicitly constructed by the algorithm for computing the Hough transform [3].In many cases an edge detector can be used as a pre-processing stage to obtain image points or image pixels that are on the desired curve in the image space. Due to imperfections in either the image data or the edge detector, however, there may be missing points or pixels on the desired curves as well as spatial deviations between the ideal line/circle/ellipse and the noisy edge points as they are obtained from the edge detector. Due to this, it is non-trivial to group the extracted edge features to an appropriate set of lines, circles or ellipses. The Hough transform is used to group the edge points into object candidates by performing an explicit voting procedure over a set of parameterized image objects.Hough transform is a linear transform for detecting straight lines. In the image space, the straight line can be described as $y=m x+b$ and can be plotted for each pair of image points (x, y). In the Hough transform the characteristics of the straight line is considered not as image points, instead, in terms of its polar coordinate pair, denoted $r$ and $\Theta$ (theta).The parameter $r$ represents the distance between the line and the origin, while $\Theta$ is the angle of the vector from the origin to this closest point. Using this parameterization, the equation of the line can be written as

$$
y=\left(-\frac{\cos \theta}{\sin \theta}\right) x+\left(\frac{r}{\sin \theta}\right)
$$

Which can be rearranged to

$$
r=x \cos \theta+y \sin \theta
$$

It is therefore possible to associate with each line of the image a pair $(\mathrm{r}, \theta)$ which is unique if $\theta \in[0, \pi)$ and $r \in \mathbf{R}$, or if $\theta \in[0,2 \pi)_{\text {and }} r \geq 0$.

The $(r, \theta)$ plane is sometimes referred to as Hough space for the set of straight lines in two dimensions. This corresponds to a sinusoidal curve in the $(r, \theta)$ plane, which is unique to that point. If the curves corresponding to two points are superimposed, the location (in the Hough space) where they cross corresponds to a line (in the original image space) that passes through both points. The Hough transform algorithm uses an array, called an accumulator to detect the existence of a line. Two unknown parameters $(r, \theta)$ is the dimension of the accumulator array and it would correspond to quantized values for $(r, \theta)$. For each pixel and its neighborhood, the Hough transform algorithm determines if there is enough evidence of an edge at that pixel. If so, it will calculate the parameters of that line, and then look for the accumulator's bin that the parameters fall into, and increase the value of that bin. By finding the bins with the highest values, typically by looking for local maxima in the accumulator space, the most likely lines can be extracted, and theirapproximate geometric definitions read off. The simplest way of finding these peaks is by applying some form of threshold determining which lines are found as well as how many. Since the lines returned do not contain any length information, it is often necessary to find which parts of the image match up with which lines. The result of the Hough transform is stored in a matrix that often is called an accumulator. One dimension of this matrix are the angles $\theta$ and the other dimension are the distances $r$, and each element has a value telling how many points/pixels are positioned on the line with parameters $(r, \theta)$. So the element with the highest value tells what line that is most represented in the input image.

Hough peaks Identifies peaks in Hough transform.By performing the Hough transform the radius and theta values and the Hough peaks are calculated. The $r$ and theta values of these Hough peaks are extracted into two matrices and the pattern recognition is done on these values. The Hough peaks are shown graphically in Fig.3.

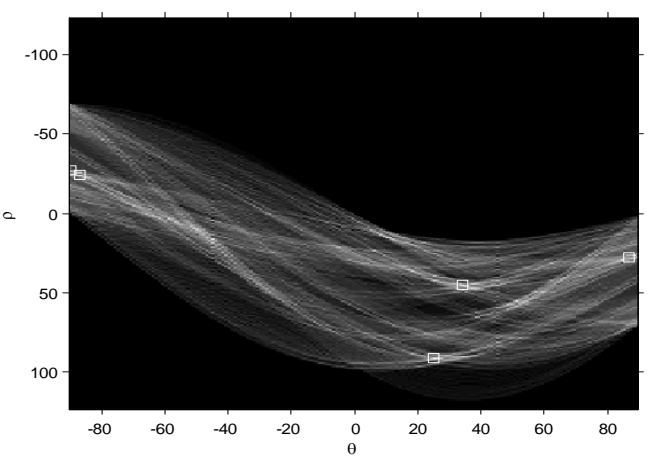

Fig.3. The Hough peaks which are represented by the small squares.

\subsection{Recognition Procedure: KNN Search}

KNNsearch is the nearest neighbor search in spaces with small intrinsic dimension. k-nearest neighbor search identifies the top $\mathrm{k}$ nearest neighbors to the query. This technique is commonly used in predictive analytics to estimate or classify a point based on the consensus of its neighbors. k-nearest neighbor graphs are graphs in which every point is connected to its $\mathrm{k}$ nearest neighbors. In those cases, we can use an algorithm which doesn't guarantee to return the actual nearest neighbor in every case, in return for improved speed or memory savings. Often such an algorithm will find the nearest neighbor in a majority of cases, but this depends strongly on the dataset being queried.Algorithms that support the approximate nearest neighbor search include locality-sensitive hashing, best bin first and balanced box-decomposition tree based search.[8] $\varepsilon^{-}$ approximate nearest neighbor search is becoming an increasingly popular tool for fighting the curse of dimensionality. We then calculate the Euclidean distances d1 and $\mathrm{d} 2$ for both $\mathrm{r}$ and $\Theta$ of test image and each and every image in the database. 


\section{RESULTSAND DISCUSSIONS}

The hand pattern images are given in Fig.4, the cropped image database is given in Fig.5, gray scaled and equalized database is given in Fig.6, thresholded database is given in Fig.7, filtered database is given in Fig.8 and thinned database is given in Fig.9. False acceptance rate and false rejection rate are two parameters considered as evaluation parameters.

\subsection{False Rejection Rate}

False rejection rate refers to the total number of authorized persons not getting access tothe system over the total number of people attempting to get the system. The false reject rate (FRR) is a measure of the probability that a biometric system will incorrectly reject an input as a negative match.We have found that out of the 80 images which are in the database, 3 images are falsely rejected. Also these 3 images are the duplicates of the ones present in the database.

Therefore,

$$
\mathrm{FRR}=(3 / 80) * 100=3.75 \%---(3)
$$

\subsection{False Acceptance Rate}

False acceptance rate (FAR) is a measure of biometric accuracy, it refers to the total number of unauthorized persons getting access to the system over the total number of people attempting to the system. It represents the probability that a given biometric system will accept an incorrect input as a positive match.We have found that out of the 20 images which are not in the database, 4 images are falsely accepted.

Therefore,

$$
\text { FAR }=(4 / 20) * 100=20 \%---(4)
$$

Which means this proposed methodology is successful with an accuracy of $96.25 \%$.Table 1 gives the Euclidean distances of images and training set, and Table 2 gives the false acceptance rate and false rejection rates.

Table 1: Euclidean distances of images and training set

\begin{tabular}{|c|c|c|c|c|c|}
\hline 10 & Imgl10_1 & 50 & 42 & Yes & Found \\
\hline 11 & Imgl11_1 & 50 & 28 & Yes & Found \\
\hline 12 & Imgl12_1 & 41 & 53 & Yes & Found \\
\hline 13 & Imgl13_1 & 31 & 60 & Yes & Found \\
\hline 14 & Imgl14 & 44 & 139 & No & Not Found \\
\hline 15 & Imgl15_1 & 60 & 41 & Yes & Found \\
\hline 16 & Imgl16_1 & 32 & 48 & Yes & Found \\
\hline 17 & Imgl17_1 & 34 & 27 & Yes & Found \\
\hline 18 & Imgl18_1 & 39 & 28 & Yes & Found \\
\hline 19 & Imgl19_1 & 116 & 68 & Yes & Not Found \\
\hline 20 & Imgl20_1 & 64 & 60 & Yes & Found \\
\hline 21 & Imgl21_1 & 56 & 77 & Yes & Found \\
\hline 22 & Imgl22_1 & 26 & 32 & Yes & Found \\
\hline 23 & Imgl23_1 & 58 & 60 & Yes & Found \\
\hline 24 & Imgl24_1 & 13 & 29 & Yes & Found \\
\hline 25 & Imgl25_1 & 29 & 68 & Yes & Found \\
\hline 26 & Imgl26_1 & 36 & 34 & Yes & Found \\
\hline 27 & Imgl27 & 39 & 17 & No & Found \\
\hline 28 & Imgl28 & 144 & 28 & No & Not Found \\
\hline 29 & Imgl29_1 & 100 & 24 & Yes & Not Found \\
\hline 30 & Imgl30_1 & 26 & 24 & Yes & Found \\
\hline 31 & Imgl31_1 & 26 & 32 & Yes & Found \\
\hline 32 & Imgl32_1 & 27 & 41 & Yes & Found \\
\hline 33 & Imgl33_1 & 54 & 35 & Yes & Found \\
\hline 34 & Imgl34 & 167 & 53 & No & Not Found \\
\hline 35 & Imgl35_1 & 59 & 23 & Yes & Found \\
\hline 36 & Imgl36 & 32 & 139 & No & Not Found \\
\hline 37 & Imgl37_1 & 11 & 17 & Yes & Found \\
\hline 38 & Imgl38 & 146 & 49 & No & Not Found \\
\hline 39 & Imgl39_1 & 25 & 73 & Yes & Found \\
\hline 40 & Imgl40 & 159 & 34 & No & Not Found \\
\hline 41 & Imgl41 & 31 & 135 & No & Not Found \\
\hline 42 & Imgl42_1 & 17 & 49 & Yes & Found \\
\hline 43 & Imgl43_1 & 58 & 37 & Yes & Found \\
\hline 44 & Imgl44 & 20 & 143 & No & Not Found \\
\hline 45 & Imgl45_1 & 49 & 77 & Yes & Found \\
\hline 46 & Imgl46_1 & 58 & 42 & Yes & Found \\
\hline 47 & Imgl47 & 152 & 42 & No & Not Found \\
\hline 48 & Imgl48_1 & 23 & 70 & Yes & Found \\
\hline 49 & Imgl49 & 30 & 127 & No & Not Found \\
\hline 50 & Imgl50 & 71 & 72 & No & Found \\
\hline 51 & Imgl51_1 & 30 & 31 & Yes & Found \\
\hline 52 & Imgl52_1 & 59 & 26 & Yes & Found \\
\hline 53 & Imgl53_1 & 22 & 50 & Yes & Found \\
\hline 54 & Imgl54 & 122 & 20 & No & Not Found \\
\hline 55 & Imgl55 & 134 & 44 & No & Not Found \\
\hline 56 & Imgl56_1 & 49 & 48 & Yes & Found \\
\hline 57 & Imgl57 & 31 & 18 & No & Found \\
\hline 58 & Imgl58 & 129 & 31 & No & Not Found \\
\hline 59 & Imgl59_1 & 47 & 35 & Yes & Found \\
\hline 60 & Imgl60_1 & 40 & 46 & Yes & Found \\
\hline 61 & Img191_1 & 24 & 56 & Yes & Found \\
\hline 62 & Imgl92 & 31 & 36 & No & Found \\
\hline 63 & Imgl93_1 & 58 & 58 & Yes & Found \\
\hline
\end{tabular}

\begin{tabular}{|l|l|l|l|l|l|}
\hline $\begin{array}{l}\text { S. } \\
\text { No. }\end{array}$ & Image No. & $\boldsymbol{d 1}$ & $\boldsymbol{d} 2$ & $\begin{array}{l}\text { In } \\
\text { Data } \\
\text { Base }\end{array}$ & $\begin{array}{l}\text { Result: Image } \\
\text { found } \text { fnot } \\
\text { found }\end{array}$ \\
\hline 1. & Imgl1_1 & 55 & 21 & Yes & Found \\
\hline 2. & Imgl2 & 169 & 152 & No & Not found \\
\hline 3. & Imgl3 & 144 & 34 & No & NotFound \\
\hline 4. & Img14_1 & 100 & 97 & Yes & Not Found \\
\hline 5. & Imgl5_1 & 86 & 37 & Yes & Found \\
\hline 6. & Imgl6_1 & 30 & 53 & Yes & Found \\
\hline 7. & Imgl7_1 & 14 & 40 & Yes & Found \\
\hline 8. & Imgl8_1 & 64 & 50 & Yes & Found \\
\hline 9. & Img19_1 & 25 & 26 & Yes & Found \\
\hline
\end{tabular}




\begin{tabular}{|l|l|l|l|l|l|}
\hline 64 & Img194_1 & 34 & 34 & Yes & Found \\
\hline 65 & Img195 & 136 & 43 & No & Not Found \\
\hline 66 & Img196_1 & 71 & 39 & Yes & Found \\
\hline 67 & Img197_1 & 75 & 34 & Yes & Found \\
\hline 68 & Img198_1 & 23 & 35 & Yes & Found \\
\hline 69 & Img199_1 & 69 & 61 & Yes & Found \\
\hline 70 & Img100_1 & 68 & 69 & Yes & Found \\
\hline 71 & Imgl1 & 0 & 0 & Yes & Found \\
\hline 72 & Img14 & 0 & 0 & Yes & Found \\
\hline 73 & Imgl5 & 0 & 0 & Yes & Found \\
\hline 74 & Img16 & 0 & 0 & Yes & Found \\
\hline 75 & Img17 & 0 & 0 & Yes & Found \\
\hline 76 & Img18 & 0 & 0 & Yes & Found \\
\hline 77 & Img19 & 0 & 0 & Yes & Found \\
\hline 78 & Imgl10 & 0 & 0 & Yes & Found \\
\hline 79 & Imgl11 & 0 & 0 & Yes & Found \\
\hline 80 & Imgl12 & 0 & 0 & Yes & Found \\
\hline 81 & Imgl13 & 0 & 0 & Yes & Found \\
\hline 82 & Imgl15 & 0 & 0 & Yes & Found \\
\hline 83 & Imgl16 & 0 & 0 & Yes & Found \\
\hline 84 & Imgl17 & 0 & 0 & Yes & Found \\
\hline 85 & Imgl18 & 0 & 0 & Yes & Found \\
\hline 86 & Imgl19 & 0 & 0 & Yes & Found \\
\hline
\end{tabular}

\begin{tabular}{|l|l|l|l|l|l|}
\hline 87 & Imgl20 & 0 & 0 & Yes & Found \\
\hline 88 & Imgl21 & 0 & 0 & Yes & Found \\
\hline 89 & Imgl22 & 0 & 0 & Yes & Found \\
\hline 90 & Imgl23 & 0 & 0 & Yes & Found \\
\hline 91 & Imgl24 & 0 & 0 & Yes & Found \\
\hline 92 & Imgl25 & 0 & 0 & Yes & Found \\
\hline 93 & Imgl26 & 0 & 0 & Yes & Found \\
\hline 94 & Imgl29 & 0 & 0 & Yes & Found \\
\hline 95 & Imgl30 & 0 & 0 & Yes & Found \\
\hline 96 & Imgl31 & 0 & 0 & Yes & Found \\
\hline 97 & Imgl32 & 0 & 0 & Yes & Found \\
\hline 98 & Imgl33 & 0 & 0 & Yes & Found \\
\hline 99 & Imgl35 & 0 & 0 & Yes & Found \\
\hline 100 & Imgl37 & 0 & 0 & Yes & Found \\
\hline
\end{tabular}

Table 2: False acceptance rate and false rejection rate

\begin{tabular}{|l|l|}
\hline Acceptance Rate & $100 \%$ \\
\hline False Acceptance Rate & $20 \%$ \\
\hline False Rejection Rate & $3.75 \%$ \\
\hline
\end{tabular}

\begin{tabular}{|c|c|c|c|c|c|c|}
\hline$[x+]$ & {$[\mathrm{M}]$} & $\mathrm{N}^{m}$ & Wy & [W] & {$[w]$} & {$\left[v_{1}\right]$} \\
\hline [w] & [w] & {$[x]$} & {$[\mathrm{N}]$} & {$[w]$} & [w] & [ere \\
\hline$[\mathrm{W}]$ & {$[v]$} & 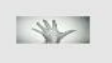 & {$[w]$} & [应] & {$[W]$} & w \\
\hline$[w]$ & [비 & {$[m]$} & [K] & [世] & WII & W \\
\hline$[\mathrm{W}]$ & W/ & Wes & [w] & [w] & {$[\mathbb{W}]$} & [w] \\
\hline 些 & WW & Ne] & we & {$\left[v_{2}\right]$} & {$[w]$} & {$\left[{ }^{\prime} \mathrm{w}=\right]$} \\
\hline [w] & सैद & $\mathrm{m}$ & [W] & {$[\underline{w}$} & [w] & image datab \\
\hline
\end{tabular}

Fig.4 Image database

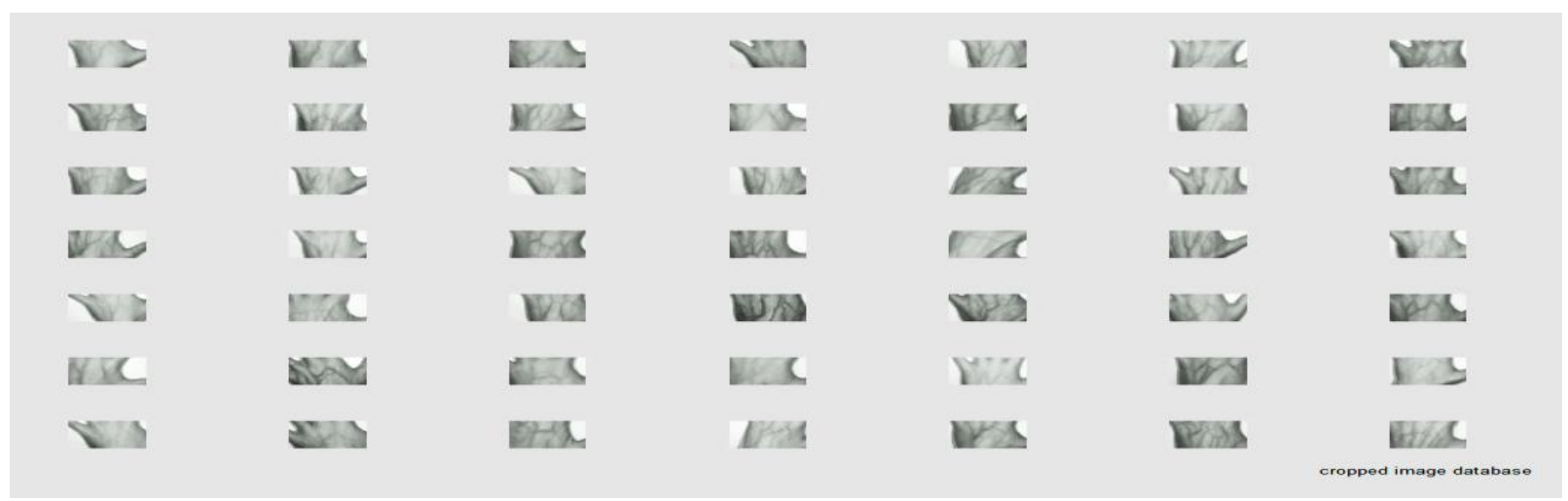

Fig.5 Cropped image database 


\begin{tabular}{|c|c|c|c|c|c|c|}
\hline$w>$ & vas & WS & XVI & VAX & $W C$ & vas \\
\hline VYs & WHA & 12 & WN & UES & $W_{H}$ & wess \\
\hline $\mathrm{W} \geqslant$ & $W \geqslant$ & Xiv & Vis: & WR & Whas & whe \\
\hline$M$ & $\mathrm{~W} / \mathrm{s}$ & Itess & HX & $A$ & WWE & $\mathrm{W} A$ \\
\hline Xi: & 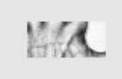 & VA & UN & WP & IXY & pas \\
\hline WiC & KXY & WS & WC & res & Wy & $1 \geq$ \\
\hline $\mathrm{W} / \mathrm{s}$ & $\mathrm{BX}$ & $H$ & D公 & IXs & Enst & HAflac \\
\hline
\end{tabular}

Fig.6 Gray-scale and adaptive histogram equalized image database

\begin{tabular}{|c|c|c|c|c|c|c|}
\hline$i \varphi$ & $M x_{t}$ & $P A$ & $\mathbf{S W}$ & $\mathbf{M}$ & 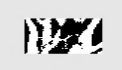 & R/4 \\
\hline M: & $\mid \mathbb{W} / !$ & IXWY & 48 & Ig: & IXI & 46 \\
\hline YYI & lot & $\mathrm{Ne}$ & MES & lirs & Nom! & W \\
\hline me & IN: & $186 !$ & W4 & $\pi$ & $W z$ & iws \\
\hline $\mathrm{NP}$ & 40 & 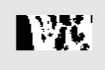 & $I^{3} \mathrm{H}^{\prime \prime}$ & WHE & akt & ! \\
\hline iare & Sap & 12x! & :MI & IX:! & ly & 17 \\
\hline Sivs: & 50 & $M$ & TKR: & 13 & Fan! & $m !$ \\
\hline
\end{tabular}

Fig.7 Threshold image database

\begin{tabular}{|c|c|c|c|c|c|c|}
\hline iv: & |YIII & ZXS & SWE & 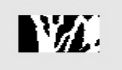 & $n$ & Rig \\
\hline W': & 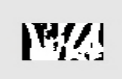 & פיני & st & Ig: & IXI & 66 \\
\hline W & $1 \mathrm{~W}$ & 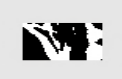 & ISW & T:PY & NW! & Na! \\
\hline ac & IM: & Yef: & 90 & $\pi$ & WE & rW: \\
\hline $\mathrm{Na}$ : & 40 & Irit & 'b5" & 17 & SBZ & ! \\
\hline vare & sent & $12 \times$ & WI & IWE! & Ir & 13 \\
\hline ives: & sos. & W! & $\mathbf{T H}$ & lW! & ping & $\begin{array}{l}W \\
\text { dimage dat }\end{array}$ \\
\hline
\end{tabular}

Fig.8 Filtered image database 


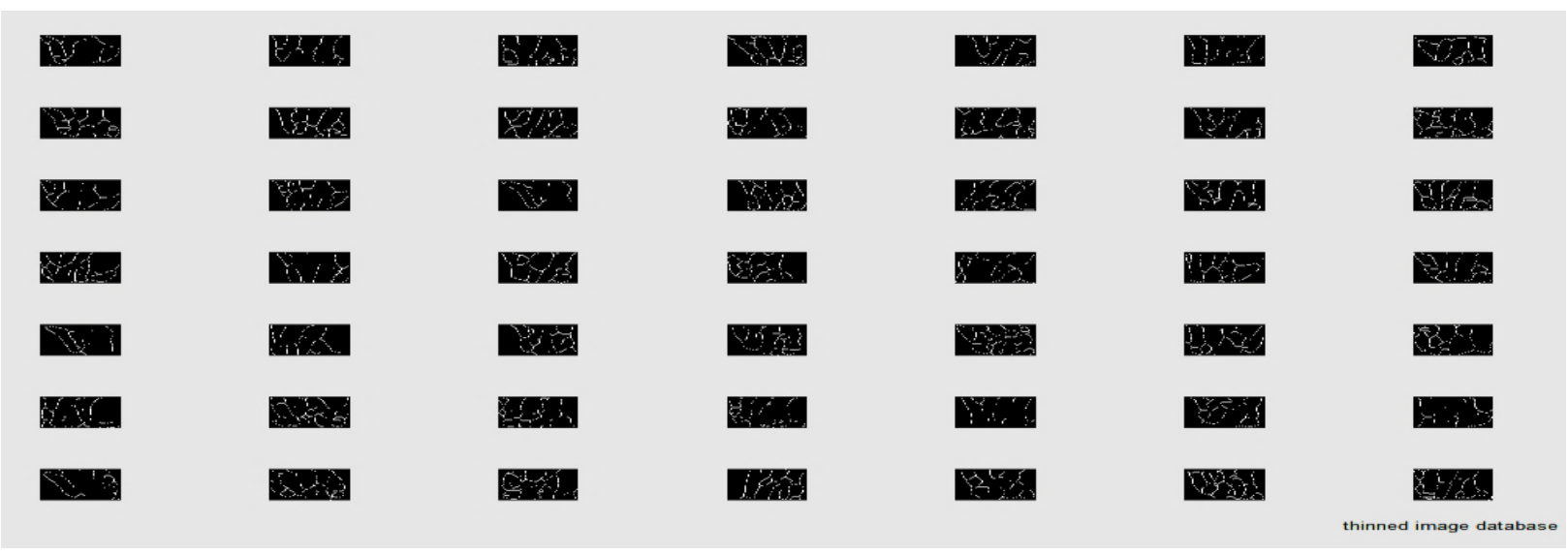

Fig.9 Thinned image database

\section{CONCLUSIONS}

The system was tested over a dataset consisting of 70 persons of different age and gender for each 2 left hand images. In this proposed work, hand vein images were pre-processed. In the pre-processing the Croppingof the images has highlighted the vein patterns. The noise filtering and thinning of the training set has removed the undesirable noise components in the database as well as query image. Next Hough transform was applied on the database to extract the features.The resulting features namely, $r$ and $\theta$ of the Hough peaks were stored in the database, which became the training set.The same procedure was applied for the query image to check for the authentication, based on the degree of similarities by using the KNN searching algorithm.We calculated the Euclidean distance between each $r$ and $\theta$ of image database and the test image. Then we found out the minimum of the value the above distances $\mathrm{d} 1$ and $\mathrm{d} 2$.Then we fixed a threshold value for these distances and authenticate the person.By following this procedure we have come up with 96.25\% accuracy with a False Acceptance rate of $20 \%$ and False rejection rate of $3.75 \%$

\section{REFERENCES}

[1] Badawi.A.M, June 26-29, 2006, "Hand Vein Biometric Verification Prototype: A Testing Performance and Patterns Similarity" In Proceedings of the 2006 International Conference on Image Processing, Computer Vision, and Pattern Recognition (IPCV'06: Las Vegas, USA,PP 3-9.

[2] Chih- Lung Lin, Kuo-Chin Fan, Feb 2004, "Biometric Verification Using Thermal Images of Palm- Dorsa Vein Patterns", IEEE Transactions on Circuits and Systems for Video Technology, VOL.14, NO.2, PP 199-213.

[3] D.H.Ballard,1981 "Generalizing the Hough transform to detect arbitrary shapes",Pattern recognition, vol.13, No.2,pp 111-122.

[4] Im.S.K, Park.H.M, Kim.S.W, Chung.C.K, and Choi.H.S, June 2000, "Improved Vein Pattern Extracting
Algorithm And Its Implementation", in Digest of technical papers of International Conference on Consumer Electronics, PP 2-3.

[5] Jain.A.K, Ross.A and Prabhakar.S, January 2004, “ An Introduction to biometric Recognition", IEEE Transactions on circuits and systems for Video Technology, Vol 14, No 1,PP 4-20.

[6] Ratha.N.K, Senior. A, and Bolle.R.M, March 2001, "Tutorial on Automated Biometrics" in Proceedings of International Conference on Advances in Pattern Recognition, Rio de Janeiro, Brazil, PP 445-474.

[7] Shi Zhao, Yiding Wang and Yunhong Wang, 2007 "Extracting Hand Vein Patterns from Low-Quality Images: A New Biometric technique Using Low-Cost Devices", IEEE, 4th International Conference on Image and Graphics,PP 667-671.

[8] Larose, D. T. (2005) k-Nearest Neighbor Algorithm, in Discovering Knowledge in Data: An Introduction to Data Mining, John Wiley \& Sons, Inc., Hoboken, NJ, USA.

[9] Tanaka.T, and Kubo.N, Aug 4-6, 2004, "Biometric Authentication by Hand Vein Patterns", SICE Annual Conference in Sapporo, PP 249-253. 